\title{
Recognition of Human Task by Attention Point Analysis
}

\section{Koichi OGAWARA Soshi IBA* Tomikazu TANUKI ${ }^{* *}$ Hiroshi KIMURA $^{* * *}$ Katsushi IKEUCHI}

Institute of Industrial Science, Univ. of Tokyo, Tokyo, 106-8558, JAPAN

*The Robotics Institute, Carnegie Mellon University, Pittsburgh PA, USA

** Research Division, Komatsu Ltd. Kanagawa, 254-8567, JAPAN

***Univ. of Electro-Communications, Tokyo, 182-8585, JAPAN

\begin{abstract}
This paper presents a novel method of constructing a human task model by attention point (AP) analysis. The AP analysis consists of two steps. At the first step, it broadly observes human task, constructs rough human task model and finds APs which require detailed analysis. Then at the second step, by applying time-consuming analysis on APs in the same human task, it can enhance the human task model. This human task model is highly abstracted and is able to change the degree of abstraction adapting to the environment so as to be applicable in a different environment.

We describe this method and its implementation using data gloves and a stereo vision system. We also show an experimental result in which a real robot observed a human task and performed the same human task successfully in a different environment using this model.
\end{abstract}

\section{Introduction}

If a robot can learn a human task through an observation and automatically increase the repertoire of its task model, it would be possible to dramatically extend the area of robot applications in a human-robot co-existent environment.

To obtain a human task, a robot must construct some human task model, which then can be applied to perform the same task, or to perform a cooperative task between a human and a robot.

So far, vision-based robot learning [1, 2] and visionbased cooperation between a human and a robot [3] have been proposed.

In these approaches, once a robot analyzes human task and constructs a human task model, the robot never turns its attention back for a closer analysis. However, it is impractical to apply detailed analysis over the entire human task to obtain a human task model, though a rough analysis over the entire human task turns out to be inadequate for parts which require detailed analysis. Therefore, we divided the analysis in two steps and proposed a novel method of constructing a human task model by attention point (AP) analysis.

\section{Attention Point (AP)}

APs, which require close observation to learn a particular behaviour, are set around specific time and position in a sequence of a human task.

We consider two kinds of APs.

I: AP for arbitration between input data separated in space

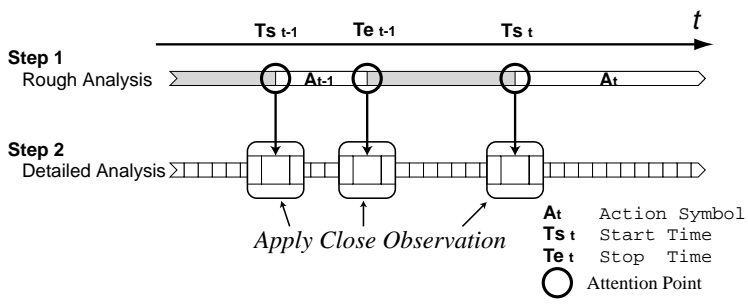

Figure 1: Two Steps Analysis using Attention Point

When several input sensors are available, it is generally ineffective to precess all the data along the entire human task. So, in this study, we determined to just record all the raw data from all the sensors along the entire human task and propose a two steps analysis of a human task(Figure $1)$. 
First, by using a minimum set of input data, a robot broadly analyzes a human task and segments the entire task into meaningful behaviours. With this analysis, the robot constructs a rough human task model and sets APs, which point to specified time and position, on boundaries of each segmented behaviour for closer analysis.

Second, by using remaining (or all) input data, the robot applies a detailed analysis at around each AP and locally enhances the human task model.

\section{II: AP for arbitration between input data separated in time}

When a robot performs the task and fails on some point, the corresponding human behaviour must be analyzed carefully, so we set APs around that point. By observing repeated human tasks and analyzing in detail around the extracted APs quantitatively, the robot can enhance the human task model.

This way, the robot can analyze the necessary parts of the human behaviour with much time and care and can build a task model efficiently. In this paper, we focus on the first AP (for arbitration between input data separated in space, Figure 1).

\section{Recognition of Human Task}

We limit the possible tasks to human hand-work on a table and describe the method of AP analysis using data gloves and stereo vision.

We can acquire depth and color images from the stereo vision and hand motion data from the data gloves. But, in this study, the image processing is much time-consuming compared to the processing of the data gloves, so we adopted two steps AP analysis to effectively handle two different input data. The robot first constructs a rough human task model using the data gloves and extracts APs. Then the robot analyzes depth and color images only around those APs to enhance the task model.

\subsection{Rough Human Task Model}

We classified possible finger actions into three actions, "Power Grasp", "Precision Grasp"[4] and "Release", and described human hand actions as a finite set of "Action Symbols" which are combinations of above finger actions and local hand motion.

So, excluding hand actions composed of independent finger motion, we can segment the entire hand-work into meaningful "Action Symbols".

As a result, we can construct a rough human task model (Figure 2) as a sequence of discrete Hand Actions which

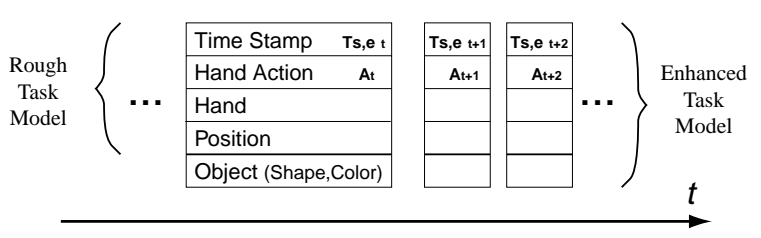

Figure 2: Human Task Model

Table 1: Attributes of Hand Action

\begin{tabular}{|l|l|l|}
\hline Attributes & Priority & Value \\
\hline \hline Time Stamp & 1 (low) & $\begin{array}{l}\text { Absolute Time } \\
\text { (start and stop time) }\end{array}$ \\
\hline Action Symbol & 3 (high) & $\begin{array}{l}\text { Power Grasp, Precision Grasp } \\
\text { Release, Pour, Hand Over }\end{array}$ \\
\hline Hand & 2 & Right, Left, Both \\
\hline Position & 1 & Absolute Position in 3D space \\
\hline Object Model & 3 & Type of the Manipulated Object \\
\hline
\end{tabular}

contain additional attributes ("Time Stamp", "Hand", "Position") as shown in Table 1.

We assigned each "Action Symbol" to a corresponding gesture and segment both hands motion by hidden markov model (HMM) based gesture spotting technique. This technique is described in chapter 4.

\subsection{Detailed Analysis around APs}

A rough task model only contains information about hand motion and knows nothing about the manipulated objects. But the model contains information about time and position of each grasping point as AP. So the robot can fetches the depth and color images which were recorded at the time of AP during observation process and applies a detailed analysis later on the specified area of the images to recognize the manipulated objects.

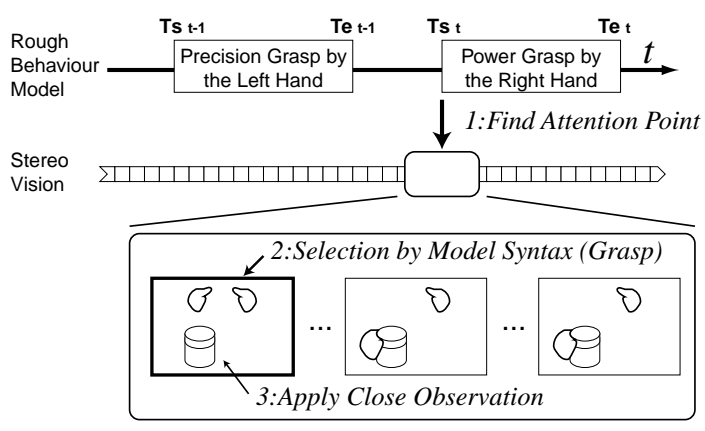

Figure 3: Example of AP Analysis 
By setting APs just before the grasping action was performed, we can get images in which the target object is not occluded by the grasping hand (Figure 3 ).

Recognition of the objects is processed by calculating shape histogram and color histogram, and the robot adds this histogram information as a new attribute "object model" into the corresponding hand action. This process is described in chapter 5 .

\subsection{Priority}

Each attribute has a priority term which tells the extent of its importance. First, the robot tries to perform the task exactly as the model tells. If it fails, the robot ignores the attributes of a lower priority for completing the task. For example, if the robot fails to perform the hand action, "Grasp the object A at the place X by the Left Hand", for some reason, it omits the attribute "Position" and "Hand" in order, and tries to grasp the object A by any hand available. Thus, by reducing the constraint before giving up the entire task, the robot can avoid discontinuance of the task.

The AP analysis constructs a human task model which is highly abstracted and is able to change the degree of abstraction adapting to the environment by the priority term. So the model can be applicable in a different environment. We show an example of this applicability in chapter 6 .

\section{Rough Analysis by Gesture Spotting}

To obtain "Action Symbol" for a rough human task model, we aimed at spotting human gestures while a human is performing some hand-works. In this study, we selected 6 gestures ( 5 described in Table $1+$ OK-sign for training) as "Action Symbols" and tried to symbolize a human task performed with the two data gloves by gesture spotting based on Hidden Markov Models (HMMs). Gestures from each hand are spotted in parallel, while two-handed gestures are spotted by combining results from both hands.

In this chapter, we will go over the general concept of a HMM, gesture spotting, and the description of the gesture spotting system.

\subsection{HMM}

HMMs are used to model a signal with variability in parameter space and time. HMMs model doubly stochastic processes, that are first-order Markov processes whose internal states are not directly observable and, thus, the term "hidden" is used. The observable output signal depends on probability distributions, fixed for each internal state. Since the model can disregard noise through a stochastic framework and it allows us to deal with the highly stochastic underlying structure of the process [5].

\subsection{Gesture Spotting}

Gesture spotting refers to the recognition and extraction of a meaningful segment corresponding to gestures from input signals that vary in both time and space. By using a gesture spotter, the user is able to interact with the system without keeping start and end of gestures in mind.

HMM-based pattern spotting is done by placing keywords modeled by HMMs and filler models in parallel in a loop. This way, the keyword can be recognized while rejecting non-keywords through filler models[6].

\subsection{Recognition using Data Gloves}

We used right and left data gloves (CyberGlove), and 6DOF position sensors (Polhemus) as input devices to perform HMM-based gesture spotting. Part of the system is based on the Hidden Markov Model Toolkit (HTK)[7].

As observable features of the HMMs, we are using 48 dimensional features per hand at time $t$. The feature vector consists of 18-dimension joint angles, $\left\{r_{1} \ldots r_{18}\right\}_{t}, 6$ dimension hand velocity, ${ }^{t-1} P_{t}={ }^{t-1}\{x$, $y, z, \alpha, \beta, \gamma\}_{t}$ which in fact is a velocity referenced from the previous hand coordinate, and differentials of the above 24 features.

\begin{tabular}{|c|c|c|}
\hline \multicolumn{3}{|c|}{ Table 2: Gesture definitions } \\
\hline Gesture & Primitives & Action \\
\hline Power Grasp & cls + sp & $\begin{array}{l}\text { Power-grasp from open } \\
\text { position }\end{array}$ \\
\hline Precision Grasp & $\mathrm{prc}+\mathrm{sp}$ & $\begin{array}{l}\text { Precision-grasp from } \\
\text { open position }\end{array}$ \\
\hline Pour & $\mathrm{cls}+\mathrm{roll}+\mathrm{sp}$ & $\begin{array}{l}\text { Power-grasp, and roll } \\
\text { the wrist }\end{array}$ \\
\hline Hand-over & prc + forw + sp & $\begin{array}{l}\text { Precision-grasp, move } \\
\text { forward, and back }\end{array}$ \\
\hline Release & opn+sp & Open a grasp hand \\
\hline OK-sign & $\mathrm{ok}+\mathrm{sp}$ & $\begin{array}{l}\text { Make a circle with thumb } \\
\text { and index finger }\end{array}$ \\
\hline Garbage & $\mathrm{gb}$ & A filler model for spotting \\
\hline Start,End & sil & Silence at the start and end \\
\hline
\end{tabular}

We defined a gesture as an attachment of primitive HMMs (Table 2). By sharing primitives, each gesture can use a small number of training data with better efficiency. We defined 9 primitives: cls, prc, roll, forw, opn, ok, gb, sil, sp. cls, prc, roll, forw, opn, ok are defined as 5-state leftright HMMs (models with a single way transition from the start to the end). sil is a silent state used at a time of training, $s p$ is a short pause which tends to be there at the end of the gesture, and $g b$ is a garbage collector that is trained on arbitrary non-gesture movements. 


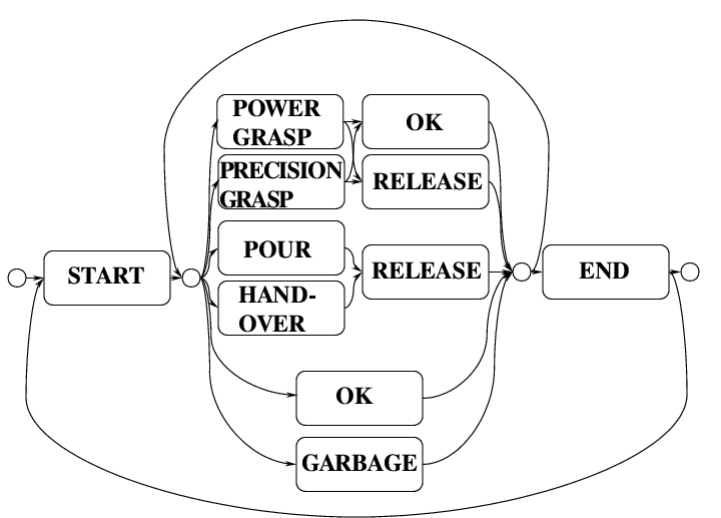

Figure 4: Gesture Transition Network

Our system can sample the data from the right and left data gloves in $30 \mathrm{~Hz}$ and spot gestures in parallel without delay. Figure 4 has information on the HMM grammar network used in gesture spotting.

\section{Detailed Analysis by Stereo Vision System}

To enhance a rough human task model, we utilize shape histogram and color histogram as an object model.

We assume that human task is demonstrated on a table whose geometric information is known. By removing the table surface from the acquired depth image, we can extract each object on the table.

Shape histogram is calculated as a list of matching likelihood between an object extracted in the depth image and each prearranged geometric object models. Matching likelihood is calculated by 3D template matching technique.

Color histogram is calculated as a normalized hue histogram which counts pixels with large saturation value among the area of the object on the color image.

These depth and color images are produced at $5 \mathrm{fps}$ (up to $30 \mathrm{fps}$ ) by 9eye multi-baseline stereo vision system[8].

In this chapter, we describe 3D Template Matching (3DTM) technique and describe the method of calculating shape and color histogram. Then we show histogram based object recognition method.

\subsection{D Template Matching (3DTM)}

3D Template Matching (3DTM)[9] is the technique to find the precise position and orientation of the target object in a depth data by projecting the corresponding 3D model.

It assumes that the 3D geometric model (template) of a target object and the initial position of the target object is known in advance. It projects the $3 \mathrm{D}$ model into the

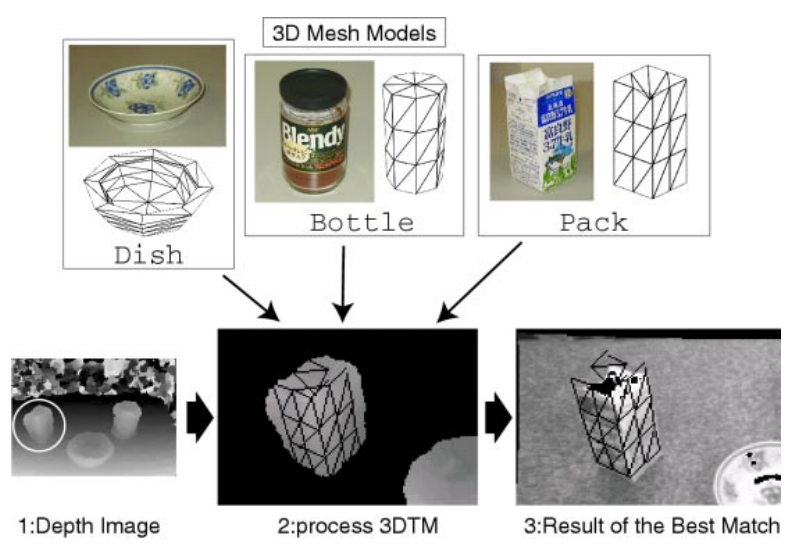

Figure 5: Recognition of objects with 3DTM

3D space generated from a depth image. Then it calculates matching likelihood between the 3D model and the 3D data by summing up weighted distance between each vertex in the template model and the closest 3D point. We adopted M-estimator which is a generalized least squared method as a weight function.

3DTM iteratively moves the model in 6D parameter space (position and orientation) to decrease the distance until it converges. The settled parameters are the estimated position and orientation.

\subsection{Shape Histogram}

The robot builds shape histogram as follows. (i) It extracts regions corresponding to objects by removing the background and the table surface from the depth data. (ii) It applies 3DTM on the extracted region using a set of known geometric models (Figure 5) and calculates matching likelihood.

Table 3: Detected Shape Histogram (Result of 3DTM)

\begin{tabular}{|c|c|c|r|}
\hline Objects & \multicolumn{3}{|c|}{ Models } \\
\cline { 2 - 4 } (in Depth Data) & Pack & Dish & Bottle \\
\hline \hline Pack Histogram & $\underline{0.25}$ & 1.30 & 0.55 \\
\hline Dish Histogram & 2.08 & $\underline{0.65}$ & 1.43 \\
\hline Bottle Histogram & 0.92 & 1.20 & $\underline{0.37}$ \\
\hline
\end{tabular}

3DTM is sensitive to the initial position of the projected model and produces a better result when the target object is not occluded. From the rough human task model, the robot can select the proper depth image at the AP and get the initial position for 3DTM (from "Position" attribute).

Table 3 shows the result of 3DTM applied to the objects 
used in our experiment. Each value indicates weighted distance by M-estimator, i.e. matching likelihood, and the underlined value is the best matching result. Each row in the table corresponds to shape histogram. This result shows that the objects were correctly registered. But if some of the models have a similar shape to each other, it occasionally fails to register the correct model because of the noise contaminated depth image and the error of the initial position. Using shape histogram instead of a single resultant value is much robust in those situations.

\subsection{Color Histogram}

When the objects on the table have similar shapes, color information has great benefit to avoid miss recognition. We divided the hue space into equally separated twelve areas and find which areas each color pixel of the target object belongs to, excluding pixels of lower saturation. Then, by summing up the number of pixels for each area and normalizing the result, we can obtain color histogram.

\subsection{Histogram Based Object Recognition}

When the robot is to perform the same task after constructing a task model, it searches for objects on the table and, for each object, it calculates mean square distance between the shape and color histogram of the object on the table and those of the object in the model. The smallest value means the best matching objects. This way the robot finds the correct object described in the model.

\section{Performance and Recognition by Robot}

\subsection{Platform}

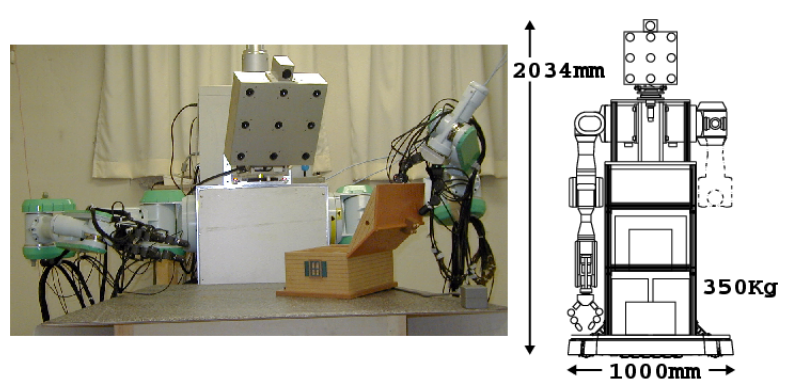

Figure 6: Platform

We have developed a robot (Figure 6) as an experimental platform for robot learning and cooperative tasks between a human and a robot. In our research, we focus on the learning and the performing of human hand-work by a robot, therefore the platform must have similar capabilities to humans, including vision, dual arms and upper body.

The main features of this robot are as follows.

- It is equipped with 9eye stereo vision system for 3D recognition (vision).

- It has dual 7DOF robot arms. The right arm has a hand with 4 fingers and the left arm has a hand with 3 fingers. Each finger has 3 DOF and a Force/Torque sensor on its tip (arms and hands).

- The robot body can freely move on 2D plane in order to move the view point and the arms in desired position (upper body).

- CORBA [10] based software architecture enables the robot to be connected easily from exterior devices such as data gloves.

\subsection{Experiment}

To examine the validity of the human task model, we set up an experiment. In this experiment, a human held the container $\mathrm{A}$ in one hand and poured the content of $\mathrm{B}$ ,which was held by the other hand, into the container A. The robot observed the task and constructed a human task model. Then the robot performed the same task in a different environment using the constructed task model.

\subsubsection{Recognition of Human Task}

First, the robot observed the human task through data gloves. As the step 1, the robot constructed a rough human task model by HMM based gesture spotter in real-time and then found APs (first part of Figure 7).

Second, the robot applied detailed analysis on the depth and color images at each AP and calculates shape and color histogram (second part of Figure 7). This analysis added the object model information to the human task model.

\subsubsection{Performance by Robot}

Then the robot performed the same task using the constructed human task model (third part of Figure 7).

To examine the applicability of this abstract human task model in a different environment, we added a new object "Dish" which was not present at the time of training, and changed the arrangement of the objects on the table.

The result shows that the robot properly chose the correct objects and completed the same task.

We performed several experiments and noticed that when the target object was out of reach of the arm described in the model, the robot omitted the "Hand" attribute whose priority is low and tried to reach the object by the other arm. This shows the effectiveness of the priority term. 


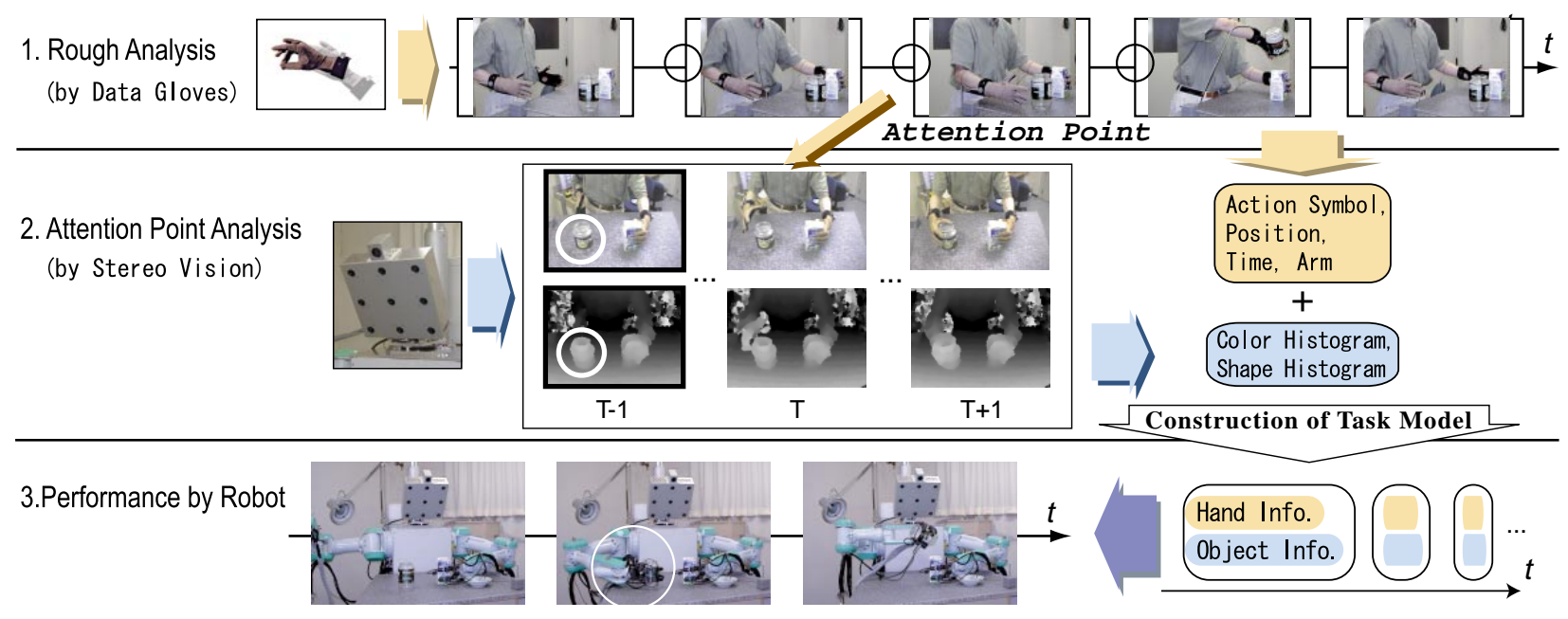

Figure 7: Experiment

The priority term changes the degree of the abstraction of each hand action adapting to the environment, so the robot can complete the task while maintaining the model description.

\section{Conclusion}

In this paper, we presented a novel method of constructing a human task model by attention point analysis using data gloves and vision system, which is also applicable in a different environment. We showed the validity of this model by an experiment, in which a real robot constructed a model of human hand-work from observation and performed the same task successfully in a different environment with this model.

\section{References}

[1] K. Ikeuchi and T. Suehiro, "Toward an Assembly Plan from Observation Part I: Task Recognition With Polyhedral Objects," IEEE Trans. Robotics and Automation, 10(3):368-384, 1994.

[2] Y. Kuniyoshi, et al., "Learning by watching," IEEE Trans. Robotics and Automation, 10(6):799-822, 1994.

[3] H. Kimura and T. Horiuchi and K. Ikeuchi, "TaskModel Based Human Robot Cooperation Using Vision,' IROS '99, 2:701-706, 1999.
[4] M. R. Cutkosky, "On Grasp Choice, Grasp Models, and the Design of Hands for Manufacturing Tasks," IEEE Trans. on Robotics and Automation, 5(3):269$279,1989$.

[5] T. Starner and A. Pentland, "Real-time American Sign Language recognition from video," IEEE International Symposium on Computer Vision, Coral Gables, FL. 265-270, 1995.

[6] K. M. Knill and S. J. Young, "Speaker Dependent Keyword Spotting for Accessing Stored Speech," Cambridge University Engineering Dept., Tech. Report, No. CUED/F-INFENT/TR 193, 1994.

[7] S. J. Young, "Hidden Markov Model Toolkit V2.2.," Entropic Research Lab Inc., Washington DC, January 1999.

[8] http://www.komatsu.co.jp/research/study56.htm

[9] M. D. Wheeler and K. Ikeuchi, "Sensor Modeling, Probabilistic Hypothesis Generation, and Robust Localization for Object Recognition", IEEE Trans. PAMI, 17(3):252-265, 1995.

[10] Common Object Request Broker Archictecture, OMG, July, 1995. 